\title{
Performance analysis of a modal converter based on an asymmetric dual-core photonic crystal fiber
}

\section{Análisis del rendimiento de un conversor modal basado en una fibra de cristal fotónico doble núcleo asimétrica}

\author{
Erick Reyes-Vera ${ }^{1,2,}{ }^{*}$, J. Úsuga ${ }^{2}$, J. Acevedo-Echeverry ${ }^{2}$, N. Gómez-Cardona², M. Varón ${ }^{1}$ \\ 1. Department of Electrical and Electronic Engineering, Universidad Nacional de Colombia, \\ A.A. 055051, Bogotá, Colombia. \\ 2. Department of Electronic and Telecommunications Engineering, Instituto Tecnológico \\ Metropolitano, A.A. 50034, Medellín, Colombia. \\ (*) E-mail: eereyesv@unal.edu.co
}

Received:01/12/2016 Accepted: 23/08/2017

DOI: $10.7149 /$ OPA.50.3.49023

\begin{abstract}
In this paper a novel modal converter based on an asymmetric dual-core photonic crystal fiber is proposed and numerically analyzed by using the full-vector finite element method. This converter allows mode conversion between the $\mathrm{LP}_{01}$ and $\mathrm{LP}_{11}$ modes, and the $\mathrm{LP}_{01}$ and $\mathrm{LP}_{21}$ modes. These modes are obtained at $1.55 \mu \mathrm{m}$. In addition, it was found that the operating wavelength of this device has a great dependence on the different geometric parameters of the structure, such as the diameter of the holes and the pitch as well. Finally, a compact device that can be used in the $\mathrm{O}+\mathrm{S}+\mathrm{C}+\mathrm{L}+\mathrm{U}$ bands with efficiency greater than $80 \%$ and a total length of $2 \mathrm{~mm}$ was obtained. This is a very interesting alternative to fabricate new all-fiber optic devices that could be implemented in mode division multiplexing systems.
\end{abstract}

Key words: Mode-division multiplexing; mode converter; photonic crystal fiber; dual-core optical fiber.

\section{RESUMEN}

En este artículo, un novedoso conversor modal basado en una fibra de cristal fotónico con núcleos asimétricos es propuesto y analizado a través de la implementación del método de elementos finitos. Este dispositivo permite la conversión modal entre los modos $\mathrm{LP}_{01}$ con el $\mathrm{LP}_{11}$ y LP $\mathrm{L}_{01}$ con el $\mathrm{LP}_{21}$ a una longitud de onda de $1.55 \mu \mathrm{m}$. Adicionalmente, hemos encontrado que la longitud de onda de operación de este tipo de dispositivos presenta una fuerte dependencia con los parámetros geométricos de la estructura tales como el diámetro de los agujeros y la distancia entre ellos. Finalmente, se obtuvo un dispositivo fotónico compacto que puede ser empleado en las bandas $\mathrm{O}+\mathrm{S}$ $+\mathrm{C}+\mathrm{L}+\mathrm{U}$ con eficiencias superiores al $80 \%$ y una longitud total de $2 \mathrm{~mm}$. Esta es una alternativa interesante a la hora de fabricar nuevos dispositivos totalmente integrados a fibra óptica que puedan ser implementados en sistemas de multiplexación por división modal.

Palabras clave: multiplexación por división modal; conversor modal; fibra de cristal fotónico; fibra óptica doble núcleo.

\section{REFERENCES AND LINKS / REFERENCIAS Y ENLACES}

[1] D. J. Richardson, J. M. Fini, and L. E. Nelson, "Space-division multiplexing in optical fibres," Nat. Photonics, vol. 7, no. April, pp. 354-362 (2013). https://doi.org/10.1038/nphoton.2013.94

[2] H. Takara, T. Takahashi, K. Nakajima, and Y. Miyamoto, "Ultra-High-Capacity Optical Transmission Using Multicore Space-Division-Multiplexing," in 18th OptoElectronics and Communications 
Conference held jointly with 2013 International Conference on Photonics in Switching (OECC/PS), pp. 2011-2012 (2013).

[3] W. Klaus, J. Sakaguchi, B. J. Puttnam, Y. Awaji, and N. Wada, "Optical technologies for space division multiplexing," in 2014 13th Workshop on Information Optics (WIO), 2, pp. 1-3 (2014). https://doi.org/10.1109/WIO.2014.6933294

[4] C. Wu et al., "Strong LP 01 and LP 11 mutual coupling conversion in a two-mode fiber Bragg grating," IEEE Photonics J., vol. 4, no. 4, pp. 1080-1086, (2012). https://doi.org/10.1109//PHOT.2012.2204735

[5] Y. Zhang, Y. Wang, S. Cai, M. Lan, S. Yu, and W. Gu, "Mode converter based on dual-core all-solid photonic bandgap fiber," Photonics Res., vol. 3, no. 5, pp. 220-223 (2015). https://doi.org/10.1364/PRJ.3.000220

[6] Yunhe Zhao, Y. Liu, Jianxiang Wen, and Tingyun Wang, "Mode converter based on the long period fiber gratings written in two mode fiber," in 2015 Opto-Electronics and Communications Conference (OECC), vol. 24, no. 6, pp. 1-3 (2015).

[7] P. Martelli, A. Gatto, P. Boffi, and M. Martinelli, "Free-space optical transmission with orbital angular momentum division multiplexing," Electron. Lett., vol. 47, no. 17, p. 972 (2011). https://doi.org/10.1049/el.2011.1766

[8] E. E. Reyes Vera, J. E. Usuga Restrepo, N. E. Gómez Cardona, and M. Varón, "Mode selective coupler based in a dual-core photonic crystal fiber with non-identical cores for spatial mode conversion," in Latin America Optics and Photonics Conference, p. LTu3C.1. (2016). https://doi.org/10.1364/LAOP.2016.LTu3C.1

[9] Y. Weng, X. He, J. Wang, and Z. Pan, "All-optical ultrafast wavelength and mode converter based on inter-modal four-wave mixing in few-mode fibers," Opt. Commun., vol. 348, pp. 7-12 (2015). https://doi.org/10.1016/i.optcom.2015.03.018

[10] T. Hellwig, T. Walbaum, and C. Fallnich, "Optically induced mode conversion in graded-index fibers using ultra-short laser pulses," Appl. Phys. B, vol. 112, no. 4, pp. 499-505 (2013). https://doi.org/10.1007/s00340-013-5645-5

[11] C. X. Shi and T. Okoshi, "Mode conversion based on the periodic coupling by a reflective fiber grating.," Opt. Lett., vol. 17, no. 23, pp. 1655-7,(1992). https://doi.org/10.1364/OL.17.001655

[12] K. Saitoh and M. Koshiba, "Numerical modeling of photonic crystal fibers," J. Light. Technol., vol. 23, no. 11 , pp. 3580-3590 (2005). https://doi.org/10.1109//LT.2005.855855

[13] J. Usuga, D. Amariles, N. Correa, E. Reyes-Vera, and N. Gomez-Cardona, "Analysis of chromatic dispersion compensator using a PCF with elliptical holes," Rev. Cuba. Fis., vol. 33, no. 1, pp. 38-41, 2016.

[14] F. Velasquez-Botero, E. Reyes-Vera, and P. Torres, "Some refractometric features of dual-core chirped microstructured optical fibers," in Proceedings of SPIE, vol. 9634, p. 963450 (2015). https://doi.org/10.1117/12.2194856

[15] E. Reyes-Vera, G. Chesini, C. M. Cordeiro, and P. Torres, "Large temperature sensitivity of birefringent side-hole photonic crystal fiber filled with Indium," in Workshop on Specialty Optical Fibers and their Applications, vol. 1, p. W3.16 (2013). https://doi.org/10.1364/WSOF.2013.W3.16

[16] A. Khaleque and H. T. Hattori, "Ultra-broadband and compact polarization splitter based on gold filled dual-core photonic crystal fiber," J. Appl. Phys., vol. 118, no. 14, p. 143101 (2015). https://doi.org/10.1063/1.4932659

[17] P. Torres, E. Reyes-Vera, A. Díez, and M. V Andrés, "Two-core transversally chirped microstructured optical fiber refractive index sensor.," Opt. Lett., vol. 39, no. 6, pp. 1593-1596 (2014). https://doi.org/10.1364/OL.39.001593

[18] S. Cai, S. Yu, M. Lan, L. Gao, S. Nie, and W. Gu, "Broadband Mode Converter Based on Photonic Crystal Fiber," IEEE Photonics Technol. Lett., vol. 27, no. 5, pp. 474-477 (2015). https://doi.org/10.1109/LPT.2014.2377495

[19] M. Y. Chen and K. S. Chiang, "Mode-Selective Characteristics of an Optical Fiber with a High-Index Core and a Photonic Bandgap Cladding," IEEE J. Sel. Top. Quantum Electron., vol. 22, no. 2, (2016). https://doi.org/10.1109//STQE.2015.2464272

[20] S. Cai, S. Yu, Y. Wang, M. Lan, L. Gao, and W. Gu, "Hybrid Dual-Core Photonic Crystal Fiber for Spatial 
Mode Conversion," IEEE Photonics Technol. Lett., vol. 28, no. 3, pp. 339-342 (2016). https://doi.org/10.1109/LPT.2015.2496165

[21] F. Bagci, "A 1x4 power-splitter based on photonic crystal Y-splitter and directional couplers," Opt. Pura Opt. Pura Apl., vol. 46, no. 3, pp. 265-273 (2013). https://doi.org/10.7149/OPA.46.3.265

[22] D. L. Lee, Electromagnetic Principles of Integrated Optics, 1st Editio. Wiley, (1986).

[23] E. Reyes-Vera, N. D. Gómez-Cardona, G. Chesini, C. M. B. Cordeiro, and P. Torres, "Temperature sensibility of the birefringence properties in side-hole photonic crystal fiber filled with Indium," Appl. Phys. Lett., vol. 105, no. 20, p. 201101 (2014). https://doi.org/10.1063/1.4902157

[24] E. Reyes-Vera and P. Torres, "Influence of filler metal on birefringent optical properties of photonic crystal fiber with integrated electrodes," J. Opt., vol. 18, no. 8, p. 85804 (2016) https://doi.org/10.1088/2040-8978/18/8/085804

[25] K. J. Park, K. Y. Song, Y. K. Kim, J. H. Lee, and B. Y. Kim, "Broadband mode division multiplexer using all-fiber mode selective couplers," Opt. Express, vol. 24, no. 4, p. 3543 (2016). https://doi.org/10.1364/OE.24.003543

\section{Introduction}

Currently, the capacity of the optical links has reached its limit, even after the use of traditional multiplexing techniques such as wavelength division multiplexing (WDM) or polarization division multiplexing (PDM). Space-Division Multiplexing (SDM) has drawn the attention of the telecommunications industry because its implementation makes it possible to increase the channel transmission capacity [1]. This technique could enhance the capacity of the channel in one or two orders of magnitude. The SDM can be implemented in two different ways. The first one uses Multi-Core Fibers (MCF), with each core as an independent channel to send the information [2], [3]. The second one is the Modal Division Multiplexing (MDM), which consists of sending coded information through the different propagation modes of a multimode fiber [4]. The correct implementation of this technique demands the design of new devices for both the control and creation of the propagated modes in an optical fiber.

Over the last years, several mechanisms for exciting and manipulating the propagation of modes in FewMode Fibers (FMFs) have been studied. Some of these techniques which are based on Long Period Fiber Grating (LPFG), Fiber Bragg Gratings (FBGs), Dual-Core Fibers (DCFs), Tapered optical fibers (TOFs) and Spatial Light Modulators (SLM) have been reported [5]-[11]. Another interesting alternative is using Photonic Crystal Fibers (PCFs). This kind of fiber offers flexibility in their designs as well as the possibility of manipulating their optical properties. The suitable optical properties depend on both the geometrical parameters and the shape of the array of holes [12]-[14]. Several reports have shown that through the implementation of the PCF, it is possible to perform multiple tasks and functions at the same time, making these fibers an interesting option to develop new devices for telecommunications and sensing applications [13], [15]-[17]. Mode Selective Coupler (MSC) based on PCF is one of the best techniques to convert the fundamental mode to higher modes, avoiding the problems that other techniques imply since the device is smaller, compact, efficient and, moreover, it allows the possibility to manipulate its behavior [8], [18], [19].

In [20], Cai et al. described a MSC based on mode coupling in a hybrid dual-core PCF with two solid cores. In one of them light is propagated through the total internal reflection while in the other one the light is propagated through the photonic band-gap. The first ring of holes around the photonic band-gap core is replaced by a material with refractive index higher than the silica. The operating wavelength of the device can be tuned by varying the refractive index of these holes. Nevertheless, the selective filled is complex for real practical applications. In [8] we proposed a tunable MSC based on a dual core PCF with non-identical cores. Wavelength Tuning in $\mathrm{S}+\mathrm{C}+\mathrm{L}+\mathrm{U}$ bands is done by changing the refractive index of the liquid in whole holes. A theoretical design of a broadband LP01↔LP02 mode converter with high conversion efficiency based on a special DCF composed by a SMF and a High Order Modes (HOF)-DCF was presented by Lin in [18].

In this work, the performance analysis of a modal converter based on an asymmetric Dual-Core Photonic Crystal Fiber (DC-PCF) is proposed. The Finite Element Method (FEM) is used to optimize the operation wavelength through energy efficiency of conversion and manipulating the main geometrical parameters of the DC-PCF. Results obtained in this study may be used to manufacture an ultra-compact device that 
enables to convert the fundamental mode into a higher mode with high conversion efficiency. The first core only allows the propagation of the fundamental mode $\left(\mathrm{LP}_{01}\right)$, while the other one allows the propagation of the $\mathrm{LP}_{01}, \mathrm{LP}_{11}$ and $\mathrm{LP}_{21}$ modes. The holes of the microstructure are filled with a liquid with a refractive index of 1.41 , that allows better energy transfer among cores, thereby while the interaction increases the coupling length decreases.

\section{Mode converter design}

The DC-PCF with non-identical cores is shown in Fig. 1. The geometrical parameters are the hole pitch $(\Lambda)$ fixed to $4 \mu \mathrm{m}$, the separation between the core centers that is $3 \Lambda$, and the fill ratio $(d / \Lambda)$ that is swept between 0.27 and 0.7. Therefore, the diameter of the holes $(d)$ has values between $1.08 \mu \mathrm{m}$ to $2.80 \mu \mathrm{m}$. The optical parameters are the background silica index, assumed to be 1.45 and the refractive index of the liquid in the microstructure holes, equal to 1.41 [8]. As the relative difference in the refractive index between the holes and the bulk silica is low, the propagated modes are weakly guided and the two cores propagate the light through total internal reflection like standard optical fibers. Based on the weakly guided theory, the right core only allows the propagation of the fundamental mode ( $\left.\mathrm{LP}_{01}\right)$, while the left core allows the propagation of the $\mathrm{LP}_{01}, \mathrm{LP}_{11}$ and $\mathrm{LP}_{21}$ modes as well.

Super-mode analysis of the asymmetric DC-PCF at $\lambda=1.55 \mu \mathrm{m}$ in order to determine the optimal conditions to couple power from excited modes in left core to excited modes in the right core was carried out. The simulation results were obtained using COMSOL Multiphysics. This analysis requires that both symmetric and anti-symmetric super-modes are excited simultaneously [19], [20]. Figures 2 (a) and 2(b) show the effective index $n_{\text {eff }}$ as a function of $d / \Lambda$, and the electric field (Ex) distribution of each excited super-mode, respectively. The modes have the following notation; super-modes $1 \& 3$ are symmetric while $2 \& 4$ are anti-symmetric. In addition, as it is predicted by [20], the symmetric supermodes have a larger effective index than the asymmetric ones in all cases. The $d / \Lambda$ parameter to determine in which region it is possible to couple the modes between the cores was changed. The coupling length was estimated using equation (1). It is defined as the distance necessary to transfer all the energy from one core to the other one [21].

$$
L_{c}=\frac{2 \pi}{\beta_{s}-\beta_{a s}}=\frac{\lambda}{2\left|n_{s}-n_{a s}\right|}
$$

$\beta_{s}$ and $\beta_{a s}$ are the propagation constants of the symmetric (s) and anti-symmetric (as) modes.

The principle of operation of a modal converter is based on the coupled mode theory [22]. In this theoretical model, each core is analyzed as an independent waveguide to avoid the perturbations caused by the presence of the other core. The effective coupling between the $\mathrm{LP}_{01}$ mode on the right core and the $\mathrm{LP}_{\mathrm{mn}}(\mathrm{m}=1,2 \& \mathrm{n}=1)$ mode on the left core requires a phase-matching condition for these two interacting modes, i.e., the modal effective indices of these two modes need to be close or equal as it is evident from the equations (2) and (3). From equation (3), it is clear that the phase matching condition is reached when the propagation constants of both modes $\left(\beta_{01}, \beta_{m n}\right)$ are equals. Then, $\delta=0$ and the maximum power transfer between the cores is achieved [22]. The values of the effective refractive index at different wavelengths of each mode were obtained using the full-vectorial FEM [12], [23], [24]. On the other hand, other supported modes are unable to be effectively excited due to the unsatisfied phase-matching condition, for example the $\mathrm{LP}_{01}$ mode on the left core.

Finally, to analyze the coupling efficiency of the device equation (2), [25] was used.

$$
\begin{gathered}
P_{\text {out }}=P_{\text {in }} \frac{k^{2}}{k^{2}+\delta^{2}} \operatorname{Sin}^{2}\left(\sqrt{k^{2}+\delta^{2} z}\right) \\
\delta=\frac{\beta_{01}-\beta_{m n}}{2}
\end{gathered}
$$

Where $P_{\text {in }}$ and $P_{\text {out }}$ are the input and coupled power, respectively, $\kappa$ is the coupling constant and $z$ is the interaction length 


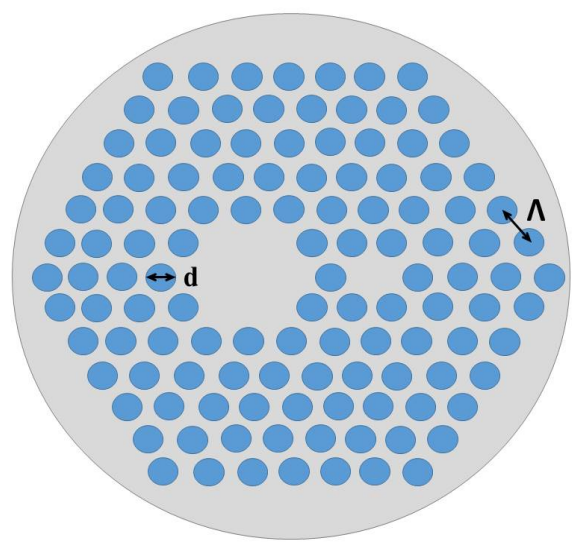

Fig.1. Schematic of asymmetric dual-core photonic crystal fiber. The grey area denotes pure silica and the blue circles denote the holes of the microstructure filled with a material of refractive index $n_{\text {Holes. }}$.

\section{Results and Discussion}

In Figure 2(a), two regions of interest can be observed. In the first region with $0.30<d / \Lambda<0.5$, the modal conversion is possible only between the $\mathrm{LP}_{01}$ of the right core and the $\mathrm{LP}_{11}$ of the left core, while in the second region $0.55<d / \Lambda<0.7$ the phenomenon occurs between $\mathrm{LP}_{01}$ mode of the right core and the $\mathrm{LP}_{21}$ mode of the left core. Furthermore, these results indicate that the average effective index of the symmetric supermodes is considerably more sensitive to variation of $d / \Lambda$ than the asymmetric supermodes. In Figure 2(b) the electric field distribution for $x$-polarization is shown. From this figure, it is clear that the supermodes $1 \& 2$ have similar distribution, and then the effective index of these modes are very similar. The same behavior occurs between the supermodes $3 \& 4$.

Figure 3(a) shows the phase matching points between the $L P_{01}$ mode of the smaller core and the $L P_{11}$ mode of the larger core for different values of the ratio $d / \Lambda$ when the wavelength is swept from $1.2 \mu \mathrm{m}$ to $2.8 \mu \mathrm{m}$. Increasing the ratio $d / \Lambda$ leads to higher values of the phase matching points, probably due to the increase of the holes diameter. When increasing the ratio $d / \Lambda$, the size of the holes is bigger and therefore the power transfer between the two cores is more difficult. Then, it is necessary to work at a larger wavelength to increase the evanescent wave in order to guarantee the energy transfer between the cores. Figure 3(b), shows the same analysis for the interaction between the $\mathrm{LP}_{01}$ mode of the smaller core and the $\mathrm{LP}_{21}$ mode of the larger core. However, in this case, the phase matching point between the modes occurs in the visible region, and it is not of interest in our analysis. The points in Figures 3 (a) and 3 (b) represent the phase-matching condition for different $d / \Lambda$.

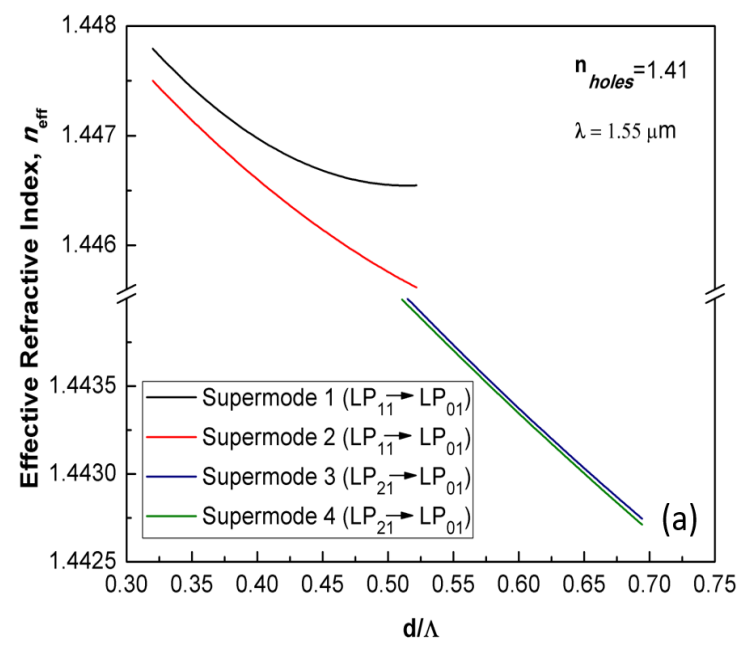

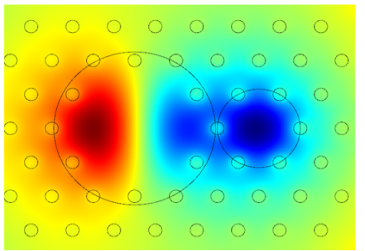

Supermode 1

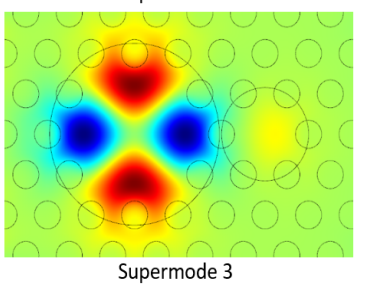

Supermode 3

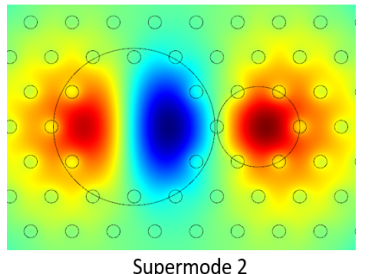

Supermode 2

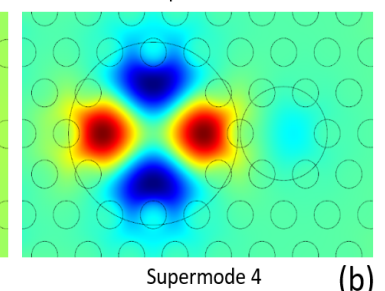

Fig. 2. (a) Supermode analysis of symmetric and asymmetric dual core PCF when the filled fraction is varied at an operating wavelength $=1.55 \mu \mathrm{m}$. (b) Electric field distribution of each supermode. 

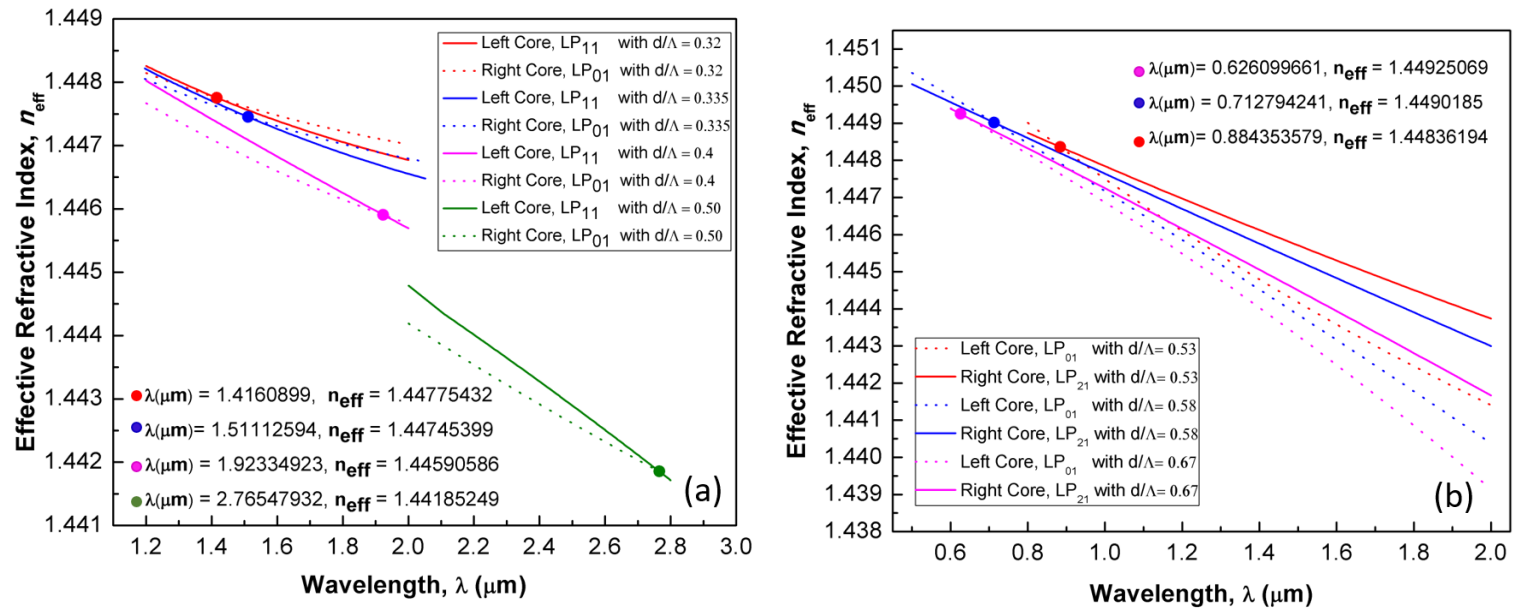

Fig. 3. Modal dispersion curves for the fundamental mode $\mathrm{LP}_{01}$ of the smaller core and (a) $\mathrm{LP}_{11}$ mode of the larger core. (b) $\mathrm{LP}_{21}$ mode of the larger core.
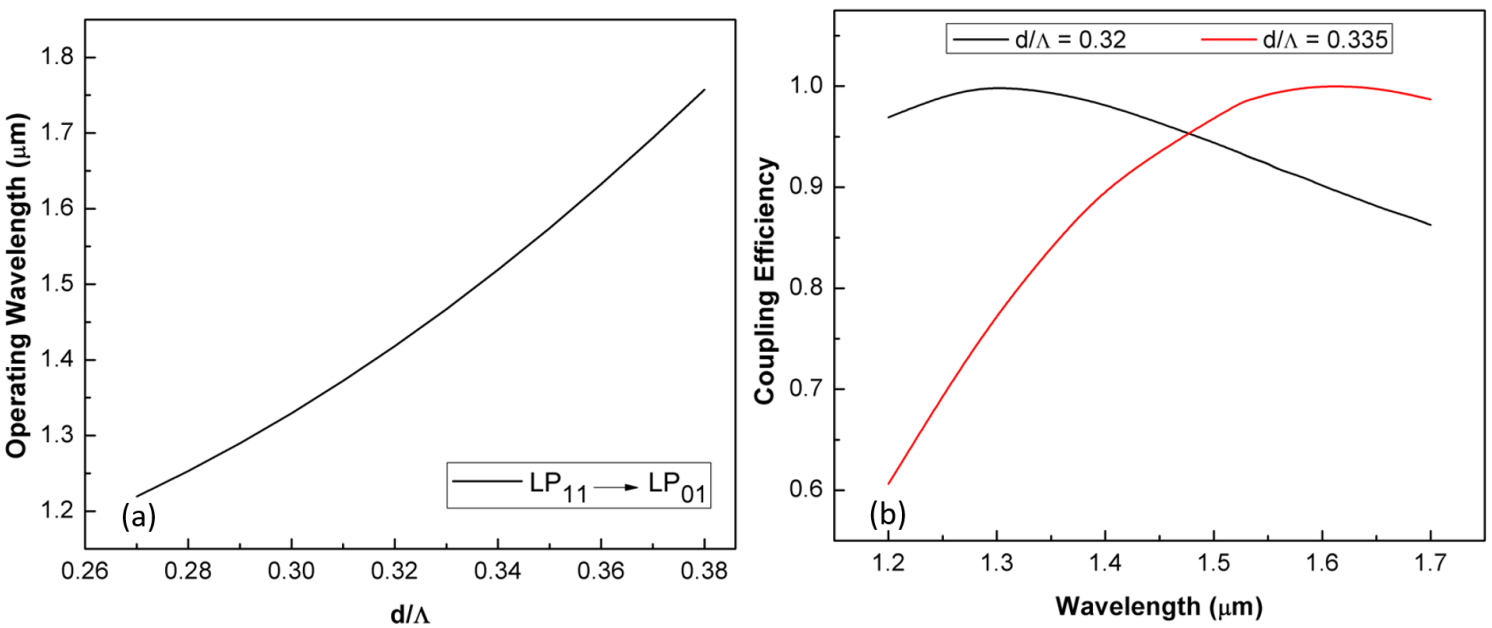

Fig. 4. (a)Operating wavelength of the device when the ratio $d / \Lambda$ increases. (b) Comparison of coupling efficiency for different configurations in the telecommunications region.

Figure 4 (a) shows the operating wavelength as a function of the ratio $d / \Lambda$. The operating wavelength in the range of interest for optical communications is presented. This limits the values of $d / \Lambda$ from 0.3 to 0.5 . The operating wavelength increases as the size of the holes increases. Greater conversion efficiency is achieved in the region close to the mismatch condition $(\Delta \beta=0)$ since in this point the effective refractive index of both modes $\mathrm{LP}_{01}$ of the right core and $\mathrm{LP}_{11}$ of the left core are the same. Operation wavelength dependence on the coupling efficiency for two values of ratio $d / \Lambda=0.320$ and 0.335 for a fixed value of $\mathrm{z}=2$ $\mathrm{mm}$ is shown in Figure 4(b). Coupling efficiencies above $60 \%$ were obtained in the $0+\mathrm{S}+\mathrm{C}+\mathrm{L}$ bands. We demonstrate that the performance of our mode-converter has a strong dependence on the geometrical parameter of the microstructure. Another interesting alternative to tune the operating wavelength of this device is the implementation of an external perturbation to change the refractive index of holes such as electric field, magnetic field, temperature or other. Results related to this alternative were presented in [8].

\section{Conclusions}

A performance analysis of a novel and ultra-compact mode converter based on an asymmetric dual-core photonic crystal fiber was proposed and analyzed using the full vectorial FEM. We demonstrate that the performance of our mode-converter has a strong dependence on the geometrical parameter of the microstructure. Through the variation of geometrical parameters, the phase matching condition can be changed and then the operating wavelength of the mode converter can be tuned. The coupling efficiency over the entire wavelength range between $1.2 \mu \mathrm{m}$ and $1.7 \mu \mathrm{m}$ was greater than $80 \%$ when the parameter 
$d / \Lambda=0.320$ and greater than $60 \%$ when $d / \Lambda=0.335$. As this device is all-fiber optic, this kind of mode selective coupler has potential applications in MDM optical fiber communications. Finally, this DC-PCF mode-converter can operate in the $\mathrm{O}+\mathrm{S}+\mathrm{C}+\mathrm{L}+\mathrm{U}$ bands.

\section{Acknowledgements}

This work was supported by the Instituto Tecnológico Metropolitano (project P15108) and Universidad Nacional de Colombia. The Instituto Tecnológico Metropolitano through the program of young researchers 2016 supported J. Úsuga. Portions of this work were presented at the IX Iberoamerican Meeting on Optics and XII Iberoamerican Meeting on Optics, Lasers and Applications (RIAO / OPTILAS), 2016. 